\title{
Quantized Transmit Beamforming With Antenna Selection in a MIMO Channel*
}

\author{
Wiroonsak Santipach \\ Department of Electrical Engineering \\ Faculty of Engineering, Kasetsart University \\ Bangkok, Thailand 10900 \\ wiroonsak.s@ku.ac.th
}

\begin{abstract}
For a point-to-point multi-input multi-output (MIMO) wireless channel, we propose a feedback scheme, which consists of transmit-antenna selection algorithm and beamforming quantization. A feedback, which is relayed from a receiver to a transmitter via a feedback channel, specifies a set of active transmit antennas and associated beamforming vector, which contains transmit antenna coefficients. Assuming perfect channel knowledge, the receiver selects the set of transmit antennas that maximizes the largest eigenvalue of a channel covariance matrix and then, chooses the beamforming vector that maximizes the capacity, from a random vector quantization (RVQ) codebook. Entries in the RVQ codebook are independent isotropically distributed and was previously shown to perform close to the optimum. We derive capacity bounds for the proposed scheme, which are functions of feedback bits, and number of active transmit antennas. The bounds are shown to approximate the actual performance well. Also complexity of the scheme can be reduced with fewer activated transmit antennas.
\end{abstract}

\section{Categories and Subject Descriptors}

H.1.1 [Models and Principles]: Systems and Information Theory

\section{General Terms}

Algorithms, Design, Theory

\section{Keywords}

Antenna selection, random vector quantization (RVQ), multiinput multi-output (MIMO), beamforming, limited feedback

\section{INTRODUCTION}

\footnotetext{
*This work was supported by Faculty of Engineering, Kasetsart University, Bangkok, Thailand, under grant 52/03/EE.
}

Permission to make digital or hard copies of all or part of this work for personal or classroom use is granted without fee provided that copies are not made or distributed for profit or commercial advantage and that copies bear this notice and the full citation on the first page. To copy otherwise, to republish, to post on servers or to redistribute to lists, requires prior specific permission and/or a fee.

IWCMC '09, June 21-24, 2009, Leipzig, Germany

Copyright 2009 ACM 978-1-60558-569-7/09/06 ...\$5.00.
Channel information at a transmitter and a receiver increases capacity of a multi-input multi-output (MIMO) fading channel [18] by allowing the transmitter to adapt its transmission to a dynamically fading channel and by helping the receiver detect transmitted symbols. A receiver can estimate channel information from a pilot signal known $a$ priori during a training period. A transmitter usually obtains channel information from a receiver via a feedback channel. Since feedback rate is normally limited, channel information has to be quantized before being sent back to the transmitter. Recent work [7,9,10,12,17] have proposed quantization schemes for a transmit beamforming vector, which consists of transmit antenna weights. Assuming perfect channel knowledge, the receiver selects the beamforming vector from a quantization codebook, which is known $a$ priori at both the transmitter and receiver, and sends the associated codebook index to the transmitter via a feedback channel. A corresponding system performance depends on a number of available feedback bits and the quantization codebook, which can be designed to maximize the capacity or minimize a symbol error rate $[6,7,9,10,12,17]$. The codebooks in all of the work mentioned require exhaustive search to locate the optimal entry. Since a number of codebook entries grows exponentially with a number of feedback bits, search complexity can be substantial and may pose a problem.

In $[4,14,20]$, another feedback scheme, which specifies a set of transmit and/or receive antennas to activate was proposed. Selected transmit antennas are allocated power to transmit signal while diversity combining on selected receive antennas is performed at the receiver [20]. With relatively few feedback bits, capacity gain with this antenna selection can be significant in some cases [14]. Also fast and efficient algorithms have been proposed for selecting antenna subsets $[3,5]$.

Here we combine a transmit beamforming quantization with a selection of transmit antennas. (This work is an extension of [17] where only quantization for the beamforming vector was considered.) The receiver, which is assumed to have perfect channel information, selects $D$ out of $N_{t}$ transmit antennas such that the corresponding channel covariance matrix gives the largest maximum eigenvalue. Then, the receiver selects the $D \times 1$ beamforming vector that maximizes the instantaneous channel capacity, from a random vector quantization (RVQ) codebook. The RVQ codebook, which contains independent isotropically distributed vectors, is simple to construct and performs close to the optimum codebook [8]. (RVQ is the optimal quantization code- 
book in a large system limit to be defined [17].) Antenna selection and the codebook index for beamforming vector are then relayed to the transmitter via an error-free feedback channel. Then, the transmitter adjusts the transmission accordingly.

Analyzing the performance of the scheme for a finite-size system is not tractable. To gain some insights, we derive bounds for a large system performance, where $N_{t}, N_{r}$ receive antennas, $D, B_{q}$ feedback bits tend to infinity with fixed ratios. We show that the large system results can predict results for a finite-size system relatively well. Numerical examples show that a gap between the derived bounds is narrow when $D$ is close to $N_{t}$. In examples shown, activating $D$ transmit antennas $\left(D<N_{t}\right)$ requires almost an order of magnitude less in search complexity than activating all transmit antennas. Other feedback schemes aimed to reduce search complexity includes $[13,15]$. The search in [13] is based on a noncoherent sequence detection algorithm while [15] uses a tree-structured codebook.

\section{CHANNEL MODEL}

We consider a discrete-time point-to-point wireless channel with $N_{t}$ transmit antennas and $N_{r}$ receive antennas. The $N_{r} \times 1$ received vector is given by

$$
\boldsymbol{y}=\boldsymbol{H} \boldsymbol{F} \boldsymbol{\alpha} b+\boldsymbol{n}
$$

where $\boldsymbol{H}=\left[h_{i j}\right]$ is an $N_{r} \times N_{t}$ channel matrix whose entry is channel coefficient $h_{i j}$ between $j$ th transmit and $i$ th receive antennas, $\boldsymbol{F}$ is an $N_{t} \times D$ matrix of basis vectors, $\boldsymbol{\alpha}$ is the $D \times 1$ beamforming vector, $b$ is a transmitted symbol with zero mean and unit variance, $\boldsymbol{n}$ is an $N_{r} \times 1$ additive white Gaussian noise vector with zero mean and variance $\sigma_{n}^{2} \boldsymbol{I}$, and $\boldsymbol{I}$ is an identity matrix. With power constraint, $\|\boldsymbol{F} \boldsymbol{\alpha}\|=1$. Assuming ideal rich scattering environment, $h_{i j}$ is a complex Gaussian random variable with zero mean and unit variance. Here we consider a rank-one transmit precoding $\boldsymbol{\alpha}$ or beamforming. An arbitrary-rank $\boldsymbol{\alpha}$ with multiple independent data streams was considered in [17].

The associated channel capacity is the maximum mutual information between $b$ and $\boldsymbol{y}$ given by

$$
C=E_{\boldsymbol{H}} \log \left(1+\rho \boldsymbol{\alpha}^{\dagger} \boldsymbol{F}^{\dagger} \boldsymbol{H}^{\dagger} \boldsymbol{H} \boldsymbol{F} \boldsymbol{\alpha}\right)
$$

where $\rho=1 / \sigma_{n}^{2}$ is the background signal-to-noise ratio (SNR). Maximizing the capacity is equivalent to maximizing the received signal power since $\log$ is a monotonically increasing function. The optimal $\boldsymbol{F} \boldsymbol{\alpha}$ that maximizes capacity is the eigenvector of $\boldsymbol{H}^{\dagger} \boldsymbol{H}$ corresponding to the maximum eigenvalue. With $B$ feedback bits, $\boldsymbol{\alpha}$ and $\boldsymbol{F}$ need to be quantized.

\section{BEAMFORMING QUANTIZATION WITH TRANSMIT ANTENNA SELECTION}

Here we describe the proposed feedback scheme, which combines antenna section algorithm and quantization of beamforming vector. The receiver first selects set of transmit antennas to activate by the algorithm to be described and then, quantizes the optimal beamforming vector, assuming perfect channel information at the receiver.

\subsection{Transmit Antenna Selection}

Let $\boldsymbol{e}_{i}$ be the $i$ th column of the $N_{t} \times N_{t}$ identity matrix. (E.g., $e_{2}=\left[\begin{array}{lllll}0 & 1 & 0 & \cdots & 0\end{array}\right]^{T}$.) Activating subset of transmit antennas is equivalent to selecting $\boldsymbol{F}$ whose columns are the corresponding unit column matrices. For example, activating transmit antennas 1 and 3 from $N_{t}=5$ corresponds to selecting $\boldsymbol{F}=\left[\begin{array}{ll}\boldsymbol{e}_{1} & \boldsymbol{e}_{3}\end{array}\right]$. Our scheme is to pick $\boldsymbol{F}$ that maximizes the largest eigenvalue of $\boldsymbol{F}^{\dagger} \boldsymbol{H}^{\dagger} \boldsymbol{H} \boldsymbol{F}$. This criterion is motivated by the fact that the received signal power $\boldsymbol{\alpha}^{\dagger} \boldsymbol{F}^{\dagger} \boldsymbol{H}^{\dagger} \boldsymbol{H} \boldsymbol{F} \boldsymbol{\alpha}$ is upper bounded by the largest eigenvalue. For a few quantization bits per transmit antenna, it was shown in [17] that the quantized beamforming vector performs close to the upper bound. Other objective functions for antenna selection include maximizing a determinant or a trace of channel covariance matrix [14].

Given $D$, we let

$$
\mathcal{F}_{D}=\left\{\boldsymbol{F}_{1}, \boldsymbol{F}_{2}, \ldots, \boldsymbol{F}_{M}\right\}
$$

denote a set of all possible $\boldsymbol{F}$ 's that correspond to selecting $D$ out of $N_{t}$ transmit antennas. Thus, $M=\left(\begin{array}{c}N_{t} \\ D\end{array}\right)$. For a given $\boldsymbol{H}$, the receiver computes the largest eigenvalue of $\boldsymbol{F}_{j}^{\dagger} \boldsymbol{H}^{\dagger} \boldsymbol{H} \boldsymbol{F}_{j}$ for each $\boldsymbol{F}_{j}$ in $\mathcal{F}_{D}$. We remark that determining the largest eigenvalue is equivalent to solving a quadratic optimization problem for which there are many efficient solutions. Thus, the receiver selects

$$
\hat{\boldsymbol{F}}=\arg \max _{\boldsymbol{F}_{j} \in \mathcal{F}}\left\{\sup _{\boldsymbol{x}} \frac{\boldsymbol{x}^{\dagger} \boldsymbol{F}_{j}^{\dagger} \boldsymbol{H}^{\dagger} \boldsymbol{H} \boldsymbol{F}_{j} \boldsymbol{x}}{\boldsymbol{x}^{\dagger} \boldsymbol{x}}\right\} .
$$

We note that the number of feedback bits required to identify $\hat{\boldsymbol{F}}$ is $B_{s}=\log _{2}(M)$.

\subsection{Quantization of Beamforming Vector}

With the chosen $\hat{\boldsymbol{F}}$, the receiver selects the beamforming vector

$$
\hat{\boldsymbol{\alpha}}=\arg \max _{\boldsymbol{\alpha}_{i} \in \mathcal{V}}\left\{\gamma\left(\boldsymbol{\alpha}_{i}\right) \triangleq \frac{1}{N_{t}} \boldsymbol{\alpha}_{i}^{\dagger} \hat{\boldsymbol{F}}^{\dagger} \boldsymbol{H}^{\dagger} \boldsymbol{H} \hat{\boldsymbol{F}} \boldsymbol{\alpha}_{i}\right\}
$$

from an RVQ codebook [16],

$$
\mathcal{V}=\left\{\boldsymbol{\alpha}_{1}, \boldsymbol{\alpha}_{2}, \ldots, \boldsymbol{\alpha}_{2^{B_{q}}}\right\}
$$

where $\boldsymbol{\alpha}_{i}$ is chosen to be an independent isotropically distributed $D \times 1$ vector with unit norm and $B_{q}$ is a number of feedback bits to quantize $\boldsymbol{\alpha}$. It is simple to verify that $\left\|\hat{\boldsymbol{F}} \boldsymbol{\alpha}_{i}\right\|=1$ for all $i$. Although an RVQ codebook is simple to construct, it is shown to perform close to the optimum codebook [8].

Since $\boldsymbol{\alpha}_{i}$ 's in an RVQ codebook are i.i.d., the corresponding received power $\gamma\left(\boldsymbol{\alpha}_{i}\right)$ 's are also i.i.d. Thus, $\gamma(\hat{\boldsymbol{\alpha}})$ is the maximum of $2^{B_{q}}$ i.i.d. random variables. We can compute the average received power as follows

$$
\hat{\gamma}=E[\gamma(\hat{\boldsymbol{\alpha}})]=2^{B_{q}} \int x\left(1-F_{\gamma}(x)\right)^{2^{B_{q}}-1} f_{\gamma}(x) \mathrm{d} x
$$

where $f_{\gamma}(\cdot)$ and $F_{\gamma}(\cdot)$ are probability density function (pdf) and cumulative distribution function (cdf) for $\gamma\left(\boldsymbol{\alpha}_{i}\right)$, respectively. The expression in $(7)$ is not tractable since $f_{\gamma}(\cdot)$ and $F_{\gamma}(\cdot)$ for arbitrary $D$ are not tractable. However, we can determine (7) for two extreme cases, i.e., $D=1$ and $D=N_{t}$.

When $D=1,(4)$ is simplified to selecting the single transmit antenna

$$
j^{*}=\arg \max _{1 \leq j \leq N_{t}} \sum_{i=1}^{N_{r}}\left|h_{i j}\right|^{2}
$$

and $\hat{\boldsymbol{\alpha}}=1$. Thus, the feedback is used only to indicate which transmit antenna is to be activated. Therefore, $B_{s}=$ $\log \left(N_{t}\right)$ and $B_{q}=0$. Since $h_{i j}$ is Gaussian, $\sum_{i=1}^{N_{r}}\left|h_{i j}\right|^{2}$ is 
Chi-square distributed. Thus, $f_{\gamma}(\cdot)$ is a pdf for the maximum of $N_{r}$ Chi-squared random variables and is given by [1]

$$
f_{\gamma}(x)=\frac{N_{t}}{\left(N_{r}-1\right) !}\left(1-\mathrm{e}^{-x} \sum_{i=0}^{N_{r}-1} \frac{x^{i}}{i !}\right)^{N_{t}-1} x^{N_{r}-1} \mathrm{e}^{-x}
$$

Substituting (9) and $B_{q}=0$ in (7) give an exact performance for $D=1$.

When $D=N_{t}$, all transmit antennas are utilized $\left(B_{s}=\right.$ $0)$ and all feedback bits are used to quantize the transmit beamforming vector. Evaluating $\hat{\gamma}$ in (7) for finite $N_{t}, N_{r}$, and $B_{q}$ is difficult. However, we can analyze the limit of $\hat{\gamma}$ as $N_{t}, N_{r}$, and $B_{q}$ tend to infinity with fixed ratios [17]. The asymptotic performance was derived in [17] and was shown to approximate the performance of a finite-size system well.

\section{PERFORMANCE BOUNDS}

Deriving (7) for general $D$ is an open problem since the eigenvalue distribution for $\frac{1}{N_{t}} \hat{\boldsymbol{F}}^{\dagger} \boldsymbol{H}^{\dagger} \boldsymbol{H} \hat{\boldsymbol{F}}$ is not known. Thus, to gain some insight on how the performance depends on system parameters e.g., $N_{t}, N_{r}, D$, and $B_{q}$, we instead analyze performance bounds.

First, we derive the lower bound on a large system performance

$$
\hat{\gamma}^{\infty} \triangleq \lim _{\left(N_{t}, N_{r}, D, B_{q}\right) \rightarrow \infty} \hat{\gamma}
$$

where we let $N_{t}, N_{r}, D$, and $B_{q}$ tend to infinity with fixed $\bar{N}_{r}=N_{r} / N_{t}, \bar{D}=D / N_{t}$, and $\bar{B}_{q}=B_{q} / N_{t}$. Randomly selecting $D$ transmit antennas gives a performance lower bound on the proposed feedback scheme. Applying results from $[2,17]$, we can derive the large system lower bound

$$
\hat{\gamma}^{\infty} \geq \hat{\gamma}_{l}^{\infty}=\lim _{\left(N_{t}, N_{r}, D, B_{q}\right) \rightarrow \infty} F_{\gamma}^{-1}\left(1-2^{-B_{q}}\right)
$$

where $F_{\gamma}^{-1}(\cdot)$ is the inverse cdf for $\gamma\left(\boldsymbol{\alpha}_{i}\right)$. Rearranging (11), we have

$$
\lim _{\substack{\left(N_{t}, N_{r}, D\right) \rightarrow \infty \\ z \rightarrow \hat{\gamma}^{\infty}}}\left[1-F_{\gamma}(z)\right]^{\frac{1}{D}}=2^{-\frac{\bar{B}_{q}}{D}} .
$$

The limit on the left-hand side of (12) can be evaluated as follows $[17]$

$$
\begin{aligned}
& \lim _{\substack{\left(N_{t}, N_{r}, D\right) \rightarrow \infty \\
z \rightarrow \hat{\gamma}^{\infty}}}\left[1-F_{\gamma}(z)\right]^{\frac{1}{D}}= \\
& \min _{0<\xi<\frac{1}{b-\hat{\gamma}^{\infty}}} \exp \left\{-\int_{a}^{b} \log \left(1+\xi\left(\hat{\gamma}^{\infty}-\lambda\right)\right) g_{\frac{1}{N_{t}} \boldsymbol{F}^{\dagger} \boldsymbol{H}^{\dagger} \boldsymbol{H} \boldsymbol{F}}(\lambda) \mathrm{d} \lambda\right\}
\end{aligned}
$$

where $g_{\frac{1}{N_{t}} \boldsymbol{F}^{\dagger} \boldsymbol{H}^{\dagger} \boldsymbol{H} \boldsymbol{F}}(\cdot)$ is an asymptotic eigenvalue density for $\frac{1}{N_{t}} \boldsymbol{F}^{\dagger} \boldsymbol{H}^{\dagger} \boldsymbol{H} \boldsymbol{F}$ and $a$ and $b$ are the associated smallest and largest eigenvalues, respectively. Reference [19] shows that the asymptotic eigenvalue distribution of $\frac{1}{N_{t}} \boldsymbol{F}^{\dagger} \boldsymbol{H}^{\dagger} \boldsymbol{H} \boldsymbol{F}$ is given by

$$
g_{\frac{1}{N_{t}} \boldsymbol{F}^{\dagger} \boldsymbol{H}^{\dagger} \boldsymbol{H} \boldsymbol{F}}(\lambda)=\frac{1}{\bar{D}}\left(1-\frac{\bar{N}_{r}}{\bar{D}}\right)^{+} \delta(\lambda)+\frac{\sqrt{(\lambda-a)(b-\lambda)}}{2 \pi \bar{D} \lambda}
$$

and

$$
a=\left(\sqrt{\bar{N}_{r}}-\sqrt{\bar{D}}\right)^{2}, \quad b=\left(\sqrt{\bar{N}_{r}}+\sqrt{\bar{D}}\right)^{2} .
$$

Similar to [17], we can evaluate the expression on the right-hand side of (13). Thus, we have the following results.
For $0 \leq \bar{B}_{q} \leq \bar{B}_{q}^{*}, \hat{\gamma}_{l}^{\infty}$ satisfies the following fixed-point equation

$$
\left(\gamma_{l}^{\infty}\right)^{\bar{N}_{r}} \mathrm{e}^{-\gamma_{l}^{\infty}}=2^{-\bar{B}_{q}}\left(\frac{\bar{N}_{r}}{\mathrm{e}}\right)^{\bar{N}_{r}}
$$

and for $\bar{B}_{q} \geq \bar{B}_{q}^{*}$

$$
\begin{aligned}
\gamma_{l}^{\infty}= & \left(\sqrt{\bar{D}}+\sqrt{\bar{N}_{r}}\right)^{2}-\bar{D} \exp \left\{\frac{1}{2} \frac{\bar{N}_{r}}{\bar{D}} \log \left(\frac{\bar{N}_{r}}{\bar{D}}\right)\right. \\
& \left.-\left(\frac{\bar{N}_{r}}{\bar{D}}-1\right) \log \left(1+\frac{\sqrt{\bar{N}_{r}}}{\sqrt{\bar{D}}}\right)+\frac{\sqrt{\bar{N}_{r}}}{\sqrt{\bar{D}}}-\frac{\bar{B}_{q}}{\bar{D}} \log (2)\right\}
\end{aligned}
$$

where

$$
\bar{B}_{q}^{*}=\frac{\bar{D}}{\log (2)}\left(\frac{\bar{N}_{r}}{\bar{D}} \log \left(\frac{\sqrt{\bar{N}_{r}}}{\sqrt{\bar{D}}+\sqrt{\bar{N}_{r}}}\right)+\frac{\sqrt{\bar{N}_{r}}}{\sqrt{\bar{D}}}\right) .
$$

When $\bar{D}=1$, the lower bound equals the large system received signal power with all transmit antennas activated. Thus, the lower bound is tight for $\bar{D}=1$.

To obtain the upper bound, we consider general precoding matrix $\boldsymbol{F}$. The optimal $\boldsymbol{F}$ that maximizes the received signal power is the $N_{t} \times D$ matrix whose columns are eigenvectors for $\frac{1}{N_{t}} \boldsymbol{H}^{\dagger} \boldsymbol{H}$ associated with the $D$ largest eigenvalues and is denoted by $\boldsymbol{F}_{u}$. Thus, the eigenvalues for $\frac{1}{N_{t}} \boldsymbol{F}_{u}^{\dagger} \boldsymbol{H}^{\dagger} \boldsymbol{H} \boldsymbol{F}_{u}$ are the $D$ largest eigenvalues for $\frac{1}{N_{t}} \boldsymbol{H}^{\dagger} \boldsymbol{H}$. Thus, the asymptotic eigenvalue density for $\frac{1}{N_{t}} \boldsymbol{F}_{u}^{\dagger} \boldsymbol{H}^{\dagger} \boldsymbol{H} \boldsymbol{F}_{u}$ is given by

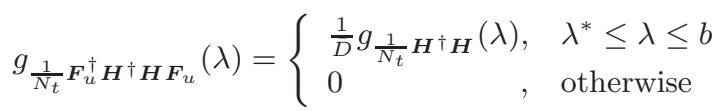

where $\lambda^{*}$ satisfies

$$
\bar{D}=\int_{\lambda^{*}}^{b} g_{\frac{1}{N_{t}} \boldsymbol{H}^{\dagger} \boldsymbol{H}}(\lambda) \mathrm{d} \lambda
$$

and $g_{\frac{1}{N_{t}} \boldsymbol{H}^{\dagger} \boldsymbol{H}}(\lambda)$ is given in (14) with $\bar{D}=1$. Substituting (19) into (13), we can solve for the asymptotic upper bound $\hat{\gamma}_{u}^{\infty}$ for given $\bar{D}$ and $\bar{B}_{q}$.

When $\bar{D}=1$, the eigenvalue distributions for $\frac{1}{N_{t}} \boldsymbol{F}_{u}^{\dagger} \boldsymbol{H}^{\dagger} \boldsymbol{H} \boldsymbol{F}_{u}$ and $\frac{1}{N_{t}} \boldsymbol{F}^{\dagger} \boldsymbol{H}^{\dagger} \boldsymbol{H} \boldsymbol{F}$ are the same. Thus, similar to the lower bound, the upper bound is tight for $\bar{D}=1$.

\section{NUMERICAL RESULTS}

In Fig. 1, we compare the capacity lower bound obtained from large system results (16)-(18) with simulation results for three different sets of parameters. The large system results are shown with lines while the simulation results are marked with + . As expected, the performance increases as normalized feedback bit $\bar{B}_{q}$ increases. The asymptotic results seem to estimate the simulation results well even with a $4 \times 4$ system. We expect the gap to narrow as system size increases.

Fig. 2 shows the upper bound for the capacity with the same set of parameters as in Fig. 1. Similarly, the performance bound increases with $\bar{B}_{q}$. For a $4 \times 4$ system, the performance shown increases by $15 \%$ with only one feedback bit per transmit antenna. Again the large system limit approximates the numerical simulation well here. Also we note that the difference between the two bounds increases as 


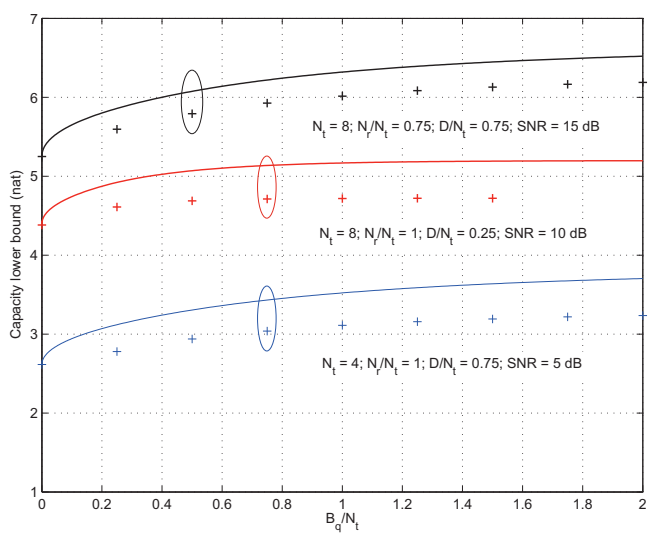

Figure 1: The lower bound on capacity is shown with $\bar{B}_{q}$ for three sets of parameters. The large system limit is shown with a solid line while the simulation result is shown with a dashed line.

$\bar{D}$ decreases. Thus, the large system bounds are more accurate in predicting the performance of the proposed scheme for a moderate to large $\bar{D}$.

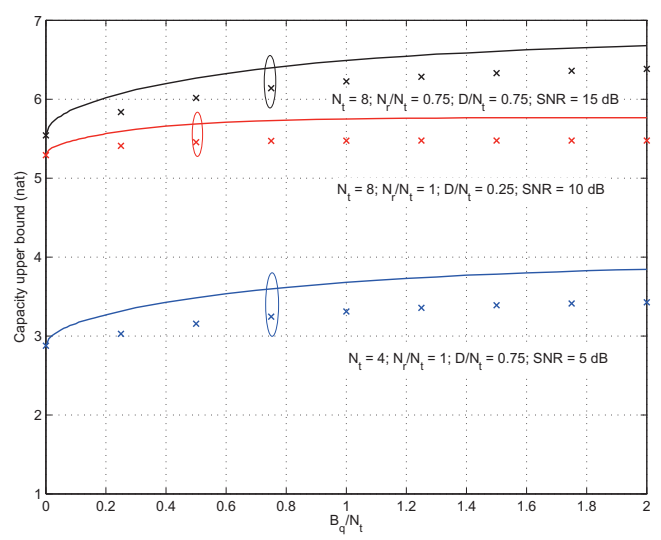

Figure 2: Similarly, the upper bound on capacity is shown with $\bar{B}_{q}$ for three sets of parameters.

We show in Fig. 3 achievable rates for the proposed scheme and both the asymptotic upper and lower bounds derived in Section 4 for two sets of parameters. For $\bar{D}=0.25$ and $\bar{D}=0.75$, the gap between the bounds are about 0.5 nat and 0.2 nat, respectively. The difference between the two bounds will be smaller for larger $\bar{D}$ and will be vanished as $\bar{D} \rightarrow 1$. Here we observe that the finite-size performance shown is not bounded by the asymptotic lower bound. As $N_{t}$ increases, the performance of the proposed scheme approaches its limit and will be bounded by the derived upper and lower bounds. Nevertheless, the two bounds are still useful in giving good approximation of the performance of the proposed feedback scheme. We note that the performance difference between no feedback and some feedback can be significant in some case, e.g., $\bar{D}=0.75$ and $\bar{B}_{q}=2$.

In Fig. 4, we plot the received power $\hat{\gamma}$ with total num-

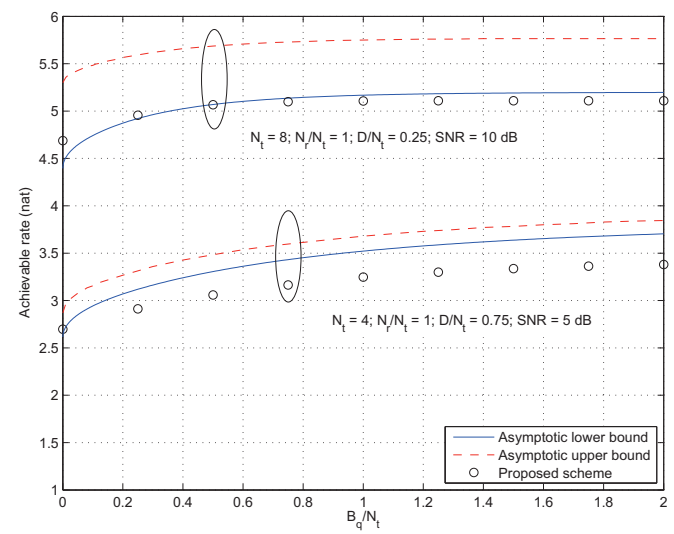

Figure 3: The achievable rate of proposed feedback scheme is shown with derived asymptotic bounds.

ber of feedback bits $B=B_{s}+B_{q}$. The simulation results shown are for $\bar{N}_{r}=0.5, N_{t}=8$, and various values of $\bar{D}$. For $\bar{D}=1$, all the transmit antennas are active and thus, all feedback is devoted to beamforming quantization. From the numerical results shown, the received power increases with total feedback bits. Since, for small $\bar{D}$, there is fewer number of beamforming coefficients to quantize, it takes relatively few feedback bits per transmit antennas to achieve the largest possible performance. (For $\bar{D}=0.25$, it takes fewer than 1.5 bits/transmit antennas.) From the figure, choosing to transmit on all antennas performs best for given total feedback bits.

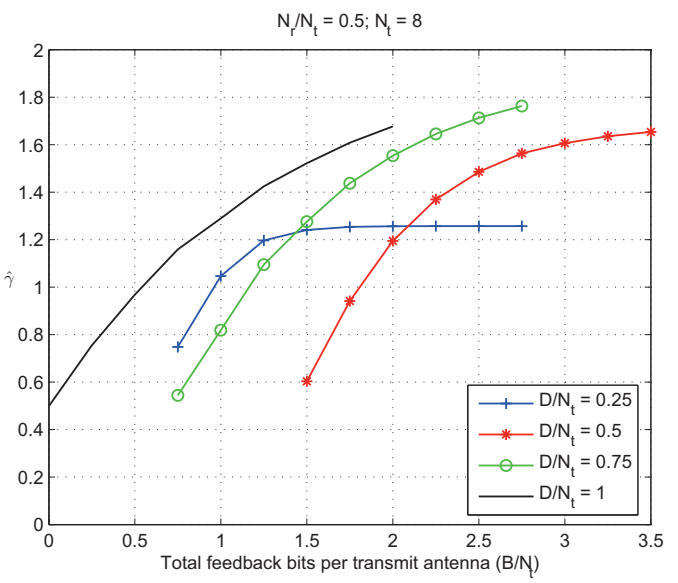

Figure 4: The received power $\hat{\gamma}$ is shown with normalized total feedback bit $\bar{B}=B / N_{t}$ for $N_{t}=8$ and $N_{r}=4$.

Besides performance, we are also concerned with complexity of the proposed scheme, which depends largely on the search algorithm to locate the quantized beamformer and set of active antennas. The search for the antenna set requires solving $\left(\begin{array}{c}N_{t} \\ D\end{array}\right)$ quadratic programs while the search for the quantized beamformer requires $2^{\bar{B}_{q} N_{t}}$ matrix computations. Since there exists a very efficient algorithm to solve quadratic program [11], we will assume that complexity of 
solving quadratic program needed in antenna selection and matrix computation needed in beamforming quantization is approximately the same. Thus, a total number of computation required is approximately $\left(\begin{array}{c}N_{t} \\ D\end{array}\right)+2^{\bar{B}_{q} N_{t}}$. Fig. 5 shows a number of total computations versus $\hat{\gamma}$ obtained from numerical simulation. For given received power, we see that selecting $\bar{D}=1$ may not give the least search complexity. For $\hat{\gamma} \approx 1.4, \bar{D}=0.5$ or activating half of transmit antennas requires the least number of computations, which is almost an order of magnitude fewer than that required for $\bar{D}=1$. For small $\hat{\gamma}$, we see that the scheme is the least complex when $\bar{D}=1$.

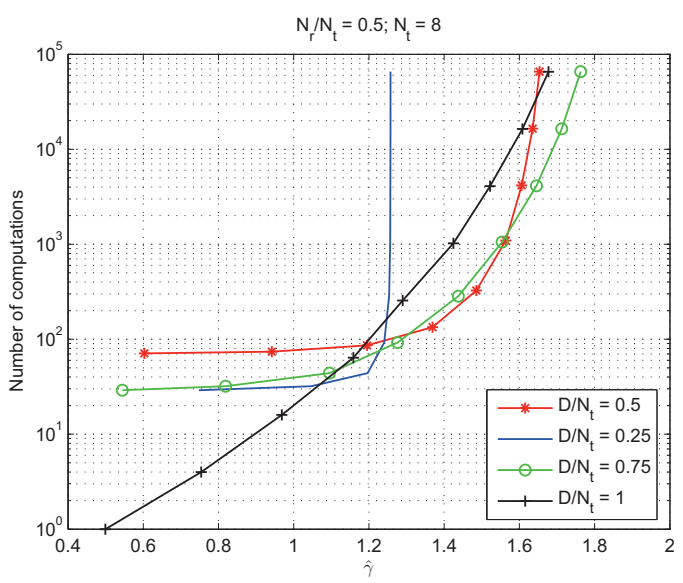

Figure 5: Shown is a number of computations required for the proposed scheme.

\section{CONCLUSIONS}

We proposed the feedback scheme, which combines beamforming quantization and transmit antenna selection. With full channel knowledge, the receiver selects the set of active transmit antennas and quantizes the associated transmit beamforming vector. We derived the upper and lower bounds for the large system performance, which are functions of available feedback bits and number of activated transmit antennas. A gap between the two bounds is small when $\bar{D}$ is large. The capacity with the proposed scheme increases with feedback bits and can be much larger than that with no feedback. Numerical example showed that selecting the right $D$ reduces number of computations needed to locate the optimal beamforming and set of selected transmit antennas.

\section{REFERENCES}

[1] Z. Chen, J. Yuan, and B. Vucetic. Analysis of transmit antenna selection/maximal-ratio combining in Rayleigh fading channels. IEEE Trans. Veh. Technol., 54(4):1312-1321, July 2005.

[2] J. Galambos. The Asymptotic Theory of Extreme Order Statistics. Robert E. Krieger, 2nd edition, 1987.

[3] M. Gharavi-Alkhansari and A. Greshman. Fast antenna selection in MIMO systems. IEEE Trans. Signal Processing, 52(2):339-347, Feb. 2003.

[4] D. A. Gore, R. W. Heath, Jr., and A. J. Paulraj. Transmit selection in spatial multiplexing systems. IEEE Commun. Lett., 6(11):491-493, Nov. 2002.
[5] A. Gorokhov, D. A. Gore, and A. J. Paulraj. Receive antenna selection for MIMO spatial multiplexing: Theory and algorithm. IEEE Trans. Signal Processing, 52(11):2796-2807, Nov. 2003.

[6] V. K. N. Lau, Y. Liu, and T.-A. Chen. On the design of MIMO block-fading channels with feedback-link capacity constraint. IEEE Trans. Commun., 52(1):62-70, Jan. 2004.

[7] D. J. Love and R. W. Heath, Jr. Grassmannian beamforming for multiple-input multiple-output wireless systems. IEEE Trans. Info. Theory, 49(10):2735-2745, Oct. 2003.

[8] D. J. Love, R. W. Heath, Jr., W. Santipach, and M. L. Honig. What is the value of limited feedback for MIMO channels? IEEE Commun. Mag., 42(10):54-59, Oct. 2004.

[9] K. K. Mukkavilli, A. Sabharwal, E. Erkip, and B. Aazhang. On beamforming with finite rate feedback in multiple antenna systems. IEEE Trans. Info. Theory, 49(10):2562-2579, Oct. 2003.

[10] A. Narula, M. J. Lopez, M. D. Trott, and G. W. Wornell. Efficient use of side information in multiple antenna data transmission over fading channels. IEEE J. Select. Areas Commun., 16(8):1423-1436, Oct. 1998.

[11] J. Nocedal and S. J. Wright. Numerical Optimization. Springer, 2nd edition, 2006.

[12] J. C. Roh and B. D. Rao. Transmit beamforming in multiple-antenna systems with finite rate feedback: A VQ-based approach. IEEE Trans. Info. Theory, 52(3):1101-1112, Mar. 2006.

[13] D. J. Ryan, I. V. L. Clarkson, I. B. Collings, D. Guo, and M. L. Honig. QAM and PSK codebooks for limited feedback MIMO beamforming. In Proc. IEEE Int. Conf. on Commun. (ICC), Glasgow, Scotland, June 2007.

[14] S. Sanayei and A. Nosratinia. Antenna selection in MIMO systems. IEEE Commun. Mag., 42(10):68-73, Oct. 2004.

[15] W. Santipach. Tree-structured Random Vector Quantization for beamforming in a multiantenna channel. In Proc. Electrical Engineering/Electronics, Computer, Telecomm. and Info. Technology Int. Conf. (ECTI-CON), volume 1, pages 325-328, Krabi, Thailand, May 2008.

[16] W. Santipach and M. L. Honig. Signature optimization for CDMA with limited feedback. IEEE Trans. Info. Theory, 51(10):3475-3492, Oct. 2005.

[17] W. Santipach and M. L. Honig. Capacity of a multiple-antenna fading channel with a quantized precoding matrix. IEEE Trans. Info. Theory, 55(3):1218-1234, Mar. 2009.

[18] İ. E. Telatar. Capacity of multi-antenna Gaussian channels. European Trans. on Telecommun., 10:585-595, Nov. 1999.

[19] A. M. Tulino and S. Verdú. Random matrix theory and wireless communications. Foundations and Trends in Communications and Information Theory, 1(1):1-182, 2004.

[20] M. Z. Win and J. H. Winters. Analysis of hybrid selection/maximal ratio combining in Rayleigh fading. IEEE Trans. Commun., 47(12):1773-1776, Dec. 1999. 